\title{
Visual Learning Tactics for Hearing Stimuli
}

\author{
Hooman Mehrani \\ Islamic Azad University of Tonekabon, Psychological Department, Mazandaran, Iran \\ ${ }^{1}$ Homan.mehrani.1976@gmail.com
}

Correspondence Author: Hooman Mehrani., Islamic Azad University of Tonekabon, Psychological Department, Mazandaran, Iran E-mail: Homan.mehrani.1976@gmail.com

\section{Received date: 11March 2019, Accepted date: 22 April 2019, Online date: 30 April 2019}

Copyright: (C) 2019 Hooman Mehrani.,. This is an open-access article distributed under the terms of the Creative Commons Attribution License, which permits unrestricted use, distribution, and reproduction in any medium, provided the original author and source are credited.

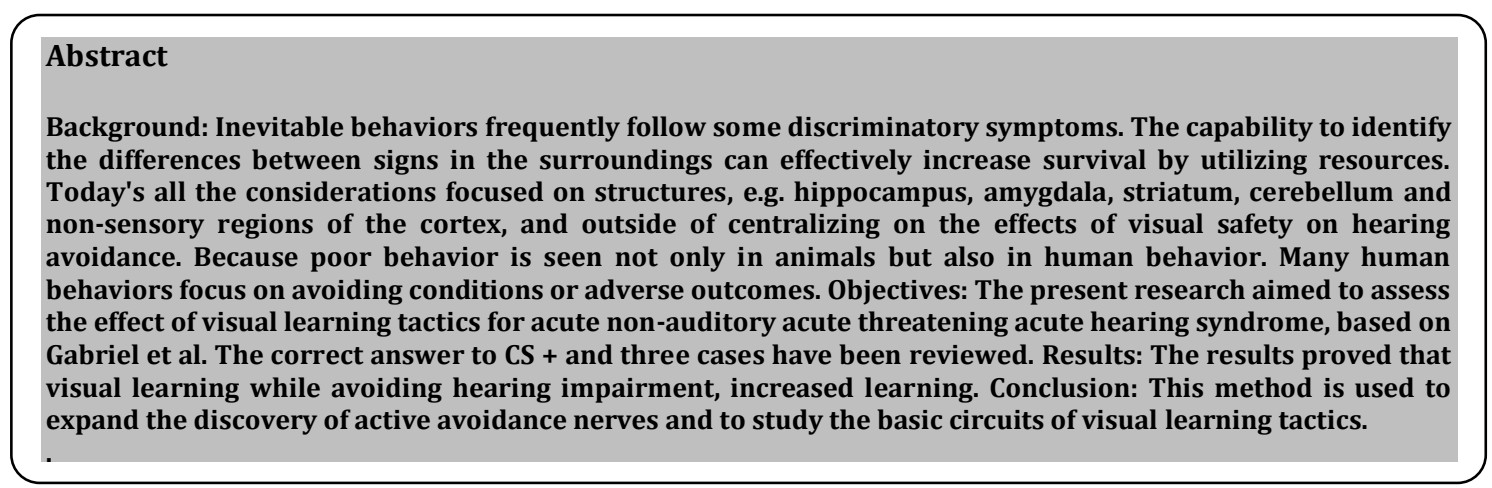

Keywords: Visual Learning, Auditory Avoidance, Avoidance Behavior, Human Behavior

\section{INTRODUCTION}

One of the most widespread paths to distinguish the use of language learning strategies is to use a preliminary ranking scale that is generally known as a questionnaire, inventory, or (less precise) survey. At this time, the most commonly used scale of strategy around the world is the inventory of strategy for language learning (SILL, Oxford, 1986-1990). Researchers prove that there could be a lot of effects in which they have likely contributed to the fact that Pavlovian ventilation has managed to make it inevitable.

Prior of all, the avoided ventilation is considered a two-step learning procedure [1-5]. In eschew of learning, the subject is first subjected to the conditions of Pavlovian ventilation and creates a connection between the shock and the signs in the device. Shock is a simple motive, and signs are conditional triggers (CS). Further, the topic learns the mechanical answer to shock eschew. After that, the "fear" created by the presence of CS strengthens the reason for learning organizational responses. Reducing fears by avoiding success is also suggested to prevent learning improvement events [4-7]. Certainly the air conditioning of Patilo is preparation for elementary prevention and also a source of "fear" in this paradigm, the study of the mechanisms of brainstorming of fear through studies of Patvovian ventilation, rather than the paradigms that the polar and the air conditioner are mixing. On the other hand, ventilation has been studied in different ways, but at that time, as it is today, it is not understood; this subtle diversity in the structure of tasks can have exhibitive factors on the brain mechanisms needed to do so. It was also less worthwhile to organize circuits in some cases such as the amygdala. Figure 1 shows the neural networks that are present in response to fear.

Therefore, some of the subterfuge researches inquired the influences of removing amygdala or numerous portions of the kidney (for review, see [8]). Finally, fear threat studies usually include a separate CS, generally one tone, that can form sensitive CSR sections to the specific amygdala nuclei that make CS processing, forming a CS-US association, and controlling the reaction Controls the defence by connecting specific engine outputs. On the other hand, probabilistic studies often contained scattered symptoms, and complex constructive responses that indirectly measured fear were not easily mapped to nerve circuits. Inevitable behaviors often follow some discriminatory symptoms. The capability to identify between different signs in the environment can effectively increase survival by applying policies. It is not suitable for animals to be protected from all environments, rather than signs that show safety and the risk can be distinguished to prevent negative stimuli and energy savings. Provides Discriminatory Threat Provides a comprehensive review of animal responses to various indications with distinct associations. Discriminative 
avoidance conditioning provides a holistic investigation of animal responses to different cues with distinct associations. Diagnostic symptoms can cause several treat mental responses and also cause differences in neurological activity [9]. Figure 2 shows the avoidance of hearing and control work. And Figure 3 indicates an avoidance response.

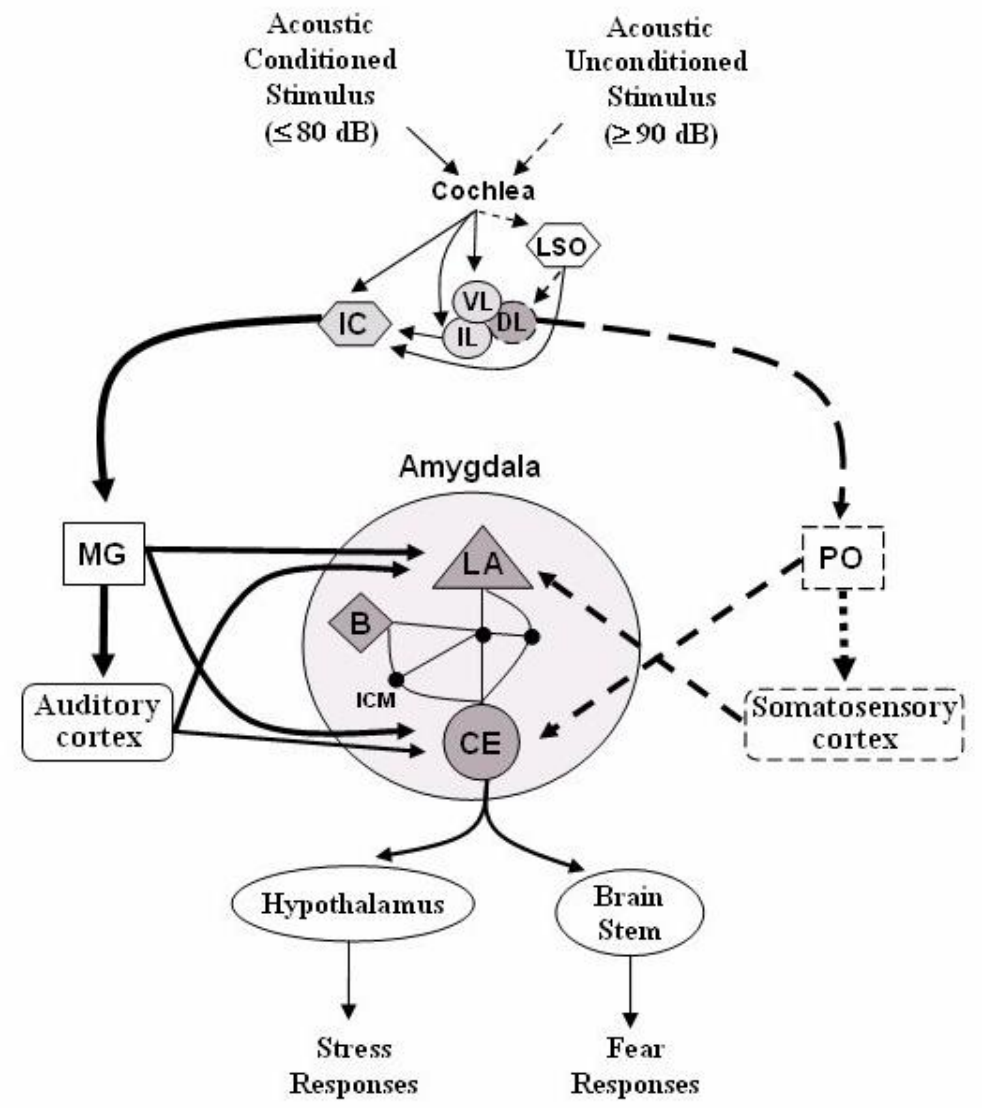

Fig. 1 The neural networks that are present in the response to fear. Hearing stimuli $(\leq 80-\mathrm{dB}, \mathrm{CS}$, permanent line $)$ are received in the ring core and sent to the lateral lens side arterial (VL) and moderate (IL) lenses directly to the IC colliculus (IC)

Avoidance Task

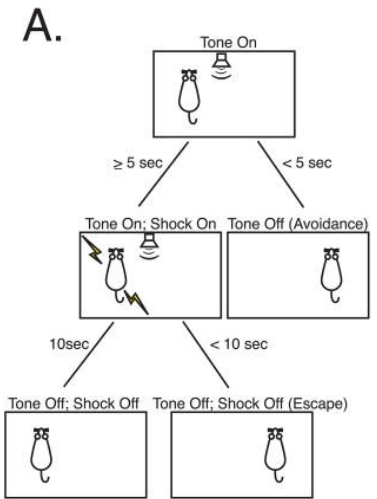

\section{Control Task}

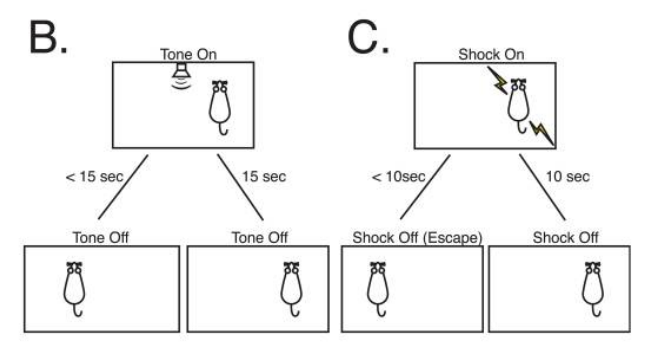

Fig.2 (A) The avoidance of hearing and control work. Adult male rats eliminate shock when they move within 5 seconds after the start of a Tone (B) control task to the opposite side of the book's shuttle. The mice received the same density and shock density that were randomly assigned to pseudo random (at least distances between stimulants $=20$ seconds).

A large volume of work by Michael Gabriel et al. (For example, $[5,10]$ ) examined neural circuits that underpin learning by using an active avoidance active threat in rabbits does is done. In their paradigm, rabbits turned to drive in response to an audio stimulus $(\mathrm{CS}+)$ displaying an electric light and ignored the presence of a different audio stimulus (CS) that did not anticipate the shock. To extend the findings of Gabriel et al., As well as answering a few questions that are not based on our own studies, including the memory of the long-term association, we wanted to create a similar paradigm in rodents. Due to differences in the behavior of the species, the riding wheels used by rabbits are not suitable for use in rodent paradigms. Research has shown the tendency for a rat to present a running wheel [11], which can inhibit learning.

A small wheel that can be run by turning the front wheels instead of a bicycle. In subterfuge ventilation, non-shock is the first amplifier, and with non-shock shading light, the light becomes a secondary amplifier. The secondary amplification was initially recognized by [12] as an actuator, which obtains the booster properties when simultaneously provided with a booster state. A number of theorists enhanced in the sense of secondary amplification, containing [6], which states that when a sign, e.g. a light 
stimulus, is generated by an individual behavior, it should be arbitrated to as a secondary amplifier. A review of the theory of two factors also includes the concept of secondary amplification, which accounts motivators in the deficiency of a negative event [12].
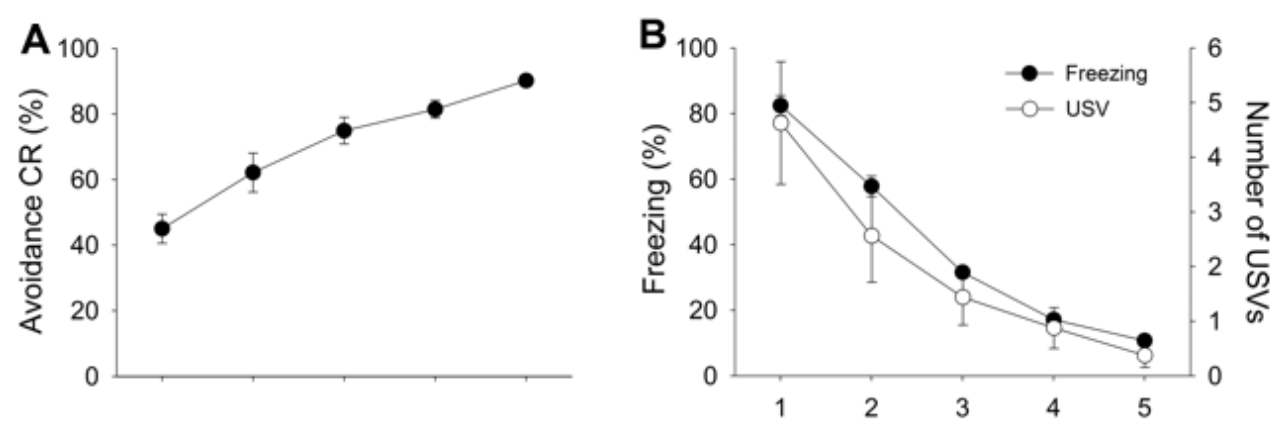

Training sessions

Fig.3 An avoidance response. The data are averaged from animals that have reached the learning standard for 5 days, respectively

These stimulants avoid the response and reduce the recall properties through fear. In pursuing a learning paradigm resolute with the repetitive learning curve, we examined the effect of adding the visual immune signal. The work was done by determining the animals that never had visual cues during exercise, animals that were displayed during their pedagogy and animals that started their pedagogy with visual cues. Ultimately, this paradigm is transformed into a trap prevention threat activity to create a more complex activity that includes memory components for creating behaviors [8]. The creation of a strong paradigm will permit farther research to provide a nervous magnetism that represents a serious threat to rodent disinfection using lesions, deactivating and recording researches. An effective example also offers the opportunity to study the circuits of the visual signal effect.

\section{THE CASE STUDIES}

To test visual learning tactics, 56 Long-Evans male rats weighing 450-650 gr. The mousse were isolated in the Spence Psychological Labs at the University of Berlin in a colony of animals with availability to food and water and was kept in a 12hour light/dark period. The three groups were organized on the basis of trial with visual immune signals: animals that never received visual signals, animals that had visual signals added during their training, and animals that started visual signal training. Animals that have been instructed in visual safety signals show a reduction day's number needed to learn, and the percentage of animals that have enhanced this. These outcomes indicate that an intrinsic immune signal enhances learning through a serious threat to hearing loss. This task is used to extend the discovery of active neural circuits and use circuits under the visual safety tactics.

All steps were in accordance with the National Institutes of Health's Instructions for the Care of Animals and experimented by the University of Berlin. The animals were instructed in a Plexiglas box $(36.5 \mathrm{~cm} \mathrm{x} 16 \mathrm{~cm} \mathrm{x} 8.6 \mathrm{~cm})$ placed in a mild sound room (Lafayette Company, $65.5 \mathrm{~cm}$ x $32.5 \mathrm{~cm} 55 \mathrm{~cm}$ ). A small red light placed the backlight in the upper left corner of the room and produced a white noise fan $(62 \mathrm{~dB})$ during the exercise. A small wheel with a diameter of $7.4 \mathrm{~cm}$ was in front of the animal. Access to half of the wheel for the animal was available to provide a behavioral response, gear movement. The animal's tail is along the $6.8-\mathrm{cm}$ propagation of Plexiglas behind the room. Using tape strips $(0.7 \mathrm{~cm}$ and $1.5 \mathrm{~cm})$, the tape was placed in two parts, approximately in a spot along the tail. Training was checked by the help of Experimentor Software. The CS + includes two tones appeared at frequencies: $3.68 \mathrm{kHz}$ and $4.286 \mathrm{kHz}$. This is almost consistent with the Notes A6 and C8. The CS also included two tones at frequencies: $0.459 \mathrm{kHz}$ and $0.562 \mathrm{kHz}$. It is almost related to notes D5 and C55, respectively on the piano. The pairs were performed to create a significant increase by creating the harmonic pair (CS +) and dissonant (CS-) (Izumi 3500). The CSs were 3 seconds, $95 \mathrm{~dB}$ in length. In addition, when an animal responded in a $2630 \mathrm{~ms}$ response window, the shock would not turn off in the same way as the main task, and roughly 1 second after the initial response of 250 lumens of bright white light (flawless lamp) to the right of the device. The light was $1 \mathrm{sec}$ and constant. This light was $6 \pm 1$ inches from the head of the animal. Figure 4 shows behavioral measurements.

Experimental parts did not improve performance with light as the only motive. Other parameters were run, the interval was between 6 and 15 seconds. There were no two cycles between enough periods to reduce the provocations before the trial began. The intervals between intervals increase up to 35 seconds to decrease the arousal constructed from the absolute stimulus prior to the onset of the subsequent trial. The test ranged from 65 experiments to 185 trials to increase the likelihood of CS + and CS presentations in sessions and to increase the parameters used in [6]. Figure 5 shows the responses of the nerve population to pure tone

\section{DATA ANALYSIS}

All offline analyzes and tests have been customized with Matlab (Mathworks, Natick, MA) and R (http://www.r-project.org/). To accurately measure the neurons showing a test frequency, we defined an index of probability encoding and called it the "Encryption Precision Index". Given that when a test frequency and a count after the stimulation of a data of the neuron X was predicted by the neuron that it was presented as a festimate, the probability (ie precision) for predicting as a festimate, $\mathrm{p}$ (festimate | fpresented), characterized as follows: 
$P\left(f_{\text {estimate }} \mid f_{\text {presented }}\right)=\sum_{x} P\left(f_{\text {estimate }} \mid x\right) P\left(x \mid f_{\text {presented }}\right)$

Where $\mathrm{p}$ (festimate $\mid \mathrm{x}$ ) is the expectancy of estimating an experiment frequency from a given spike count (that is, decoding) and $\mathrm{p}$ (x | fpresented) the expectancy of receiving the number of spike count in response to a given test (that is, encoding). Thus, equation 1 is an explanation that shows how the accuracy of the test frequency is corrupted by encryption and decryption. Both $\mathrm{p}$ (festimate $\mid x)$ and $p(x \mid$ fpresented) with a table of sets of sensors from the tune of 5 to $50 \mathrm{~ms}$ from the initiation of the excitation, $\mathrm{X}(0,1,2, \ldots)$ and a set of the test frequencies, $\mathrm{F}(1,1.3,1.6, \ldots, 32,40,50)$. This index determines the precision of the coding accuracy of the frequency display. For example, when a neuron is only active for a particular test frequency, the precision at the corresponding frequency rises.

\section{RESULTS AND DISCUSSIONS}

The reactions that were matched to CS + and CS-were computed by separating the response to the acoustic actuator with the whole number of CS + or CS within presentations in a data session. Varies scores were calculated by reducing the CS-response from the CS + response. The analysis was performed for CS + and CS-reactions, and a separate analysis was made for the varies scores.

The response delay was also analyzed by subtraction of the time of the first wheel rotation of 36.5 degrees from the start date of the CS. After that, the learning curve was analyzed for three groups of trained animals. The first group contained unexperienced animals with a visual immune signal (Without). The next group included animals that were visualized with a visual immune signal during their training (switch). The eventual group included animals that had visual immune signals during their exercises (with). Figure 6 shows the learning curve for any group. The curves were obtained with a mean of data at the spacing of 2 days. Three groups were studied: animals that never receive visual immune signals (Without), animals that switched switching to a visual immune signal, and animals that began to train with a visual immune signal (With). For Switched animals, the difference separates the exercise and indicates that the switch shows the use of the safety signal.
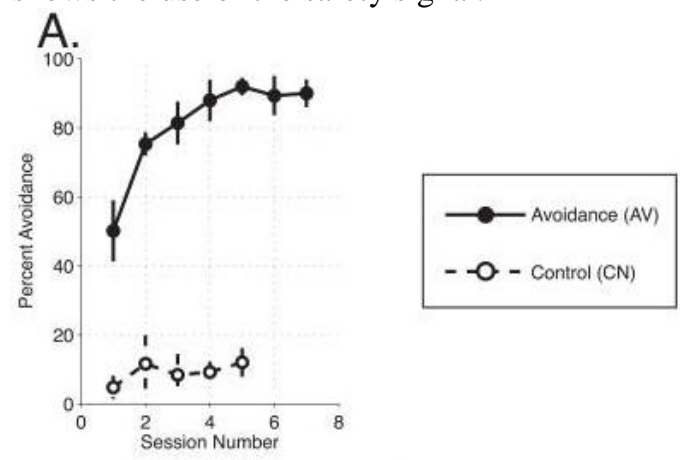

B.

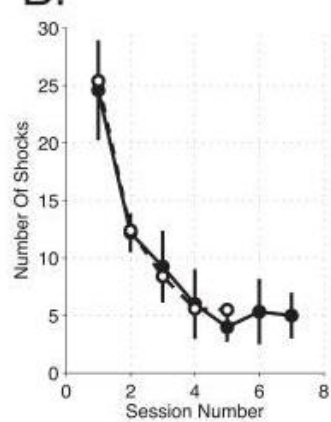

C.

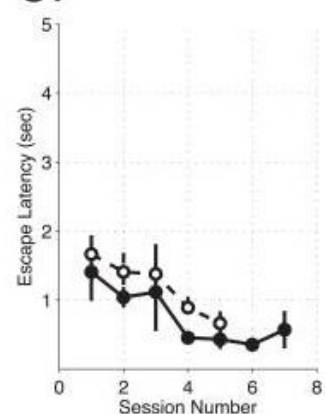

Fig. 4 Behavioral Measurements. (A) group performance. Avoid mouse showed rapid improvement during the tests sessions and obtained anomalies of $\sim 90 \%$. Given the nature of the work, control mouse were able to prevent shock. Controlling mouse did not show such learning performance. (B) Shock density. The mean number of shocks received by groups at sessions. The number of shock programs provided for controlling the mouses did not differ importantly from the inevitable group. (C) Escape from the Latency. Both groups of mouses escaped well. However, during the sessions, the escape avoidance delay was significantly shorter than the control group's delay. This vary may indicate escape responses when the mouse that are being avoided pass through the shuttle box right before the shock. 


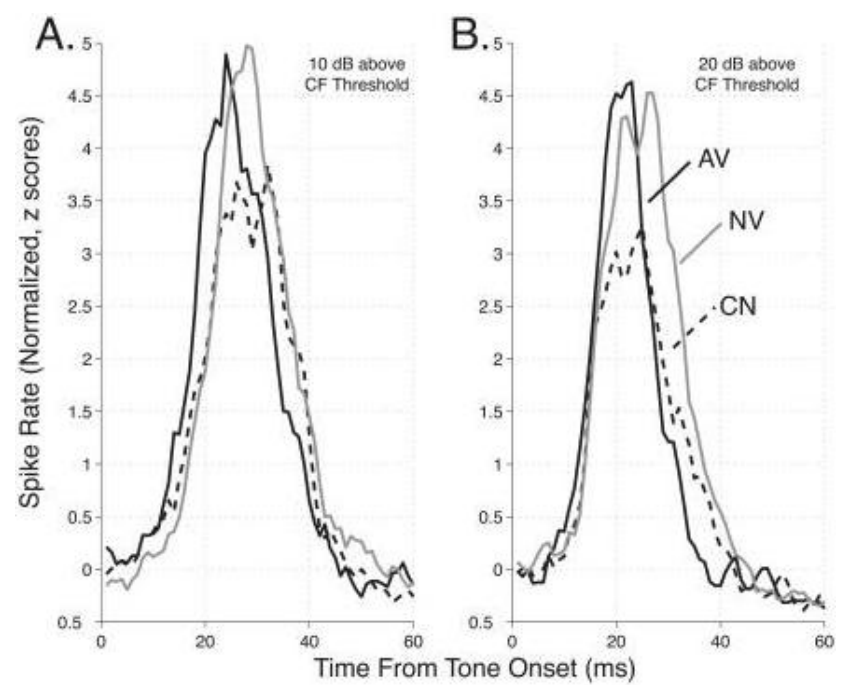

Fig. 5 Pure tone and Nervous population response. This graph shows the average of neuronal responses (with a width $=3$ milliseconds) to the tone specified at 10 $\mathrm{dB}(\mathrm{A})$ and $20 \mathrm{~dB}(\mathrm{~B})$ above the threshold of $\mathrm{CF}$.

The response for each cluster, using the average and standard deviation of the spike rate, changed after 60 milliseconds at the start of the tone. Plots indicate that the peak delay in the avoidance group ( $\mathrm{AV}=$ solid) which is superior to both simple responses (NV = gray $)$ and control $(\mathrm{CN}=$ wisdom $)$ and reduction of peak intensity in the control group. This difference between groups was controlled based on the analysis of individual clusters and in a range of stimuli.
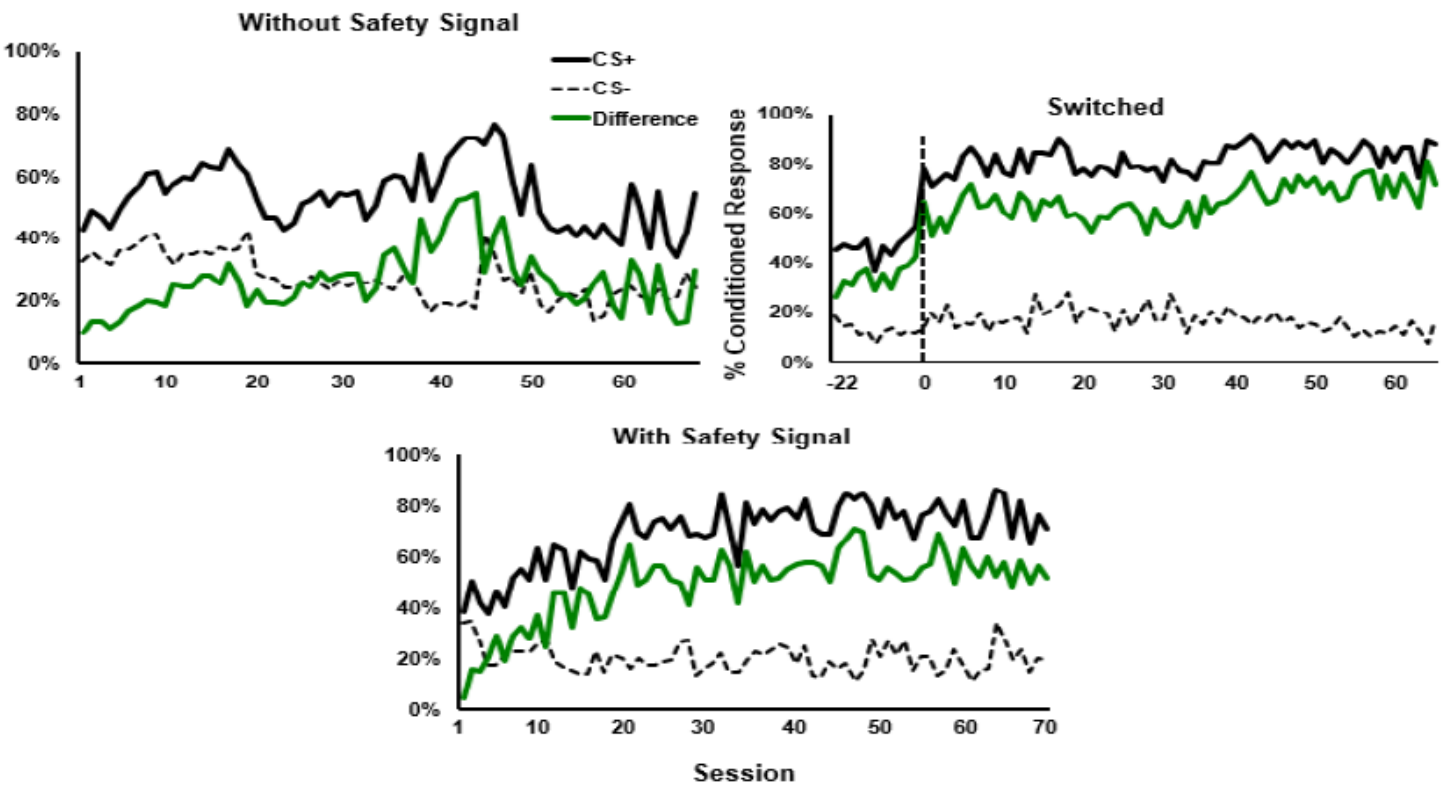

Fig. 6. Each group of learning curves

Data were analyzed using mixed-variance design analysis with a thematic variable (group: Without, switch, with), and two in-subject variables (session and CS: $\mathrm{CS}+$ and CS-) with sixty-five sessions analyzed. The main effects were found for CS $(\mathrm{F}(1.9)=264.65, \mathrm{p}<0.001)$ and session $(\mathrm{F}(63.128)=2.62, \mathrm{p}<0.001)$. The interactive influences also for group sessions $(\mathrm{F}(132.215)=2.36, \mathrm{p}<0.001)$, CS based on the group $(\mathrm{F}(2.9)=11.853 \mathrm{p}=0.002)$ and $\mathrm{CS}$ session $(\mathrm{F}(75.25)=5.27$, p \& lt; 0.001). To evaluate the effect of CS + and CS-response among groups, Pairwise values were measured using Fisher LSD test. There was no important difference between the groups for CS + , but for CS-without the group, there was considerable difference between the groups $(p=0.014)$ and the switchted group $(\mathrm{p}=0.014)$, which was close to importance in comparison with the group $(\mathrm{p}=0.033)$. There was no considerable vary between the switched groups and With $(\mathrm{p}=$ 0.329). This represents a higher response than CS for a group of animals in Switched or with a group. Figure 7 shows the influence of behavioral training on spikes 1 after pure loneliness.

When it was determined that, in addition to the visual safety signal, the performance improves, we are interested in obtaining when these behavioral changes in animals occurred in the switch conditions. We examined the first 30 first sessions of the first session that exposed the animals in the switched group to the visual immune signal. The first 20 experiments were divided into four groups of five experiments. We conducted an ANOVA research block with CS four times. An important CS effect was $\mathrm{F}$ $(1.9)=23.50, \mathrm{p}=0.012)$ but no major block effect $(\mathrm{F}(3.9)=1.13, \mathrm{p}=0.323)$ or interaction $(\mathrm{F}(1.9))=0.69, \mathrm{p}=0.630)$. Although no significant interaction has been achieved, we plan to make the first significant difference between CS + and CS on which block. This shows when animal behavior moved to a larger CS + response than CS-replication. The comparison of dispersion using LSD Block Fisher by CS showed that the first block had a significant difference between CS + and CS-block 4 ( $p=0.003$ ), which, according to experiments of 16-20 sessions with visual safety of the signal, (See figure 8). 


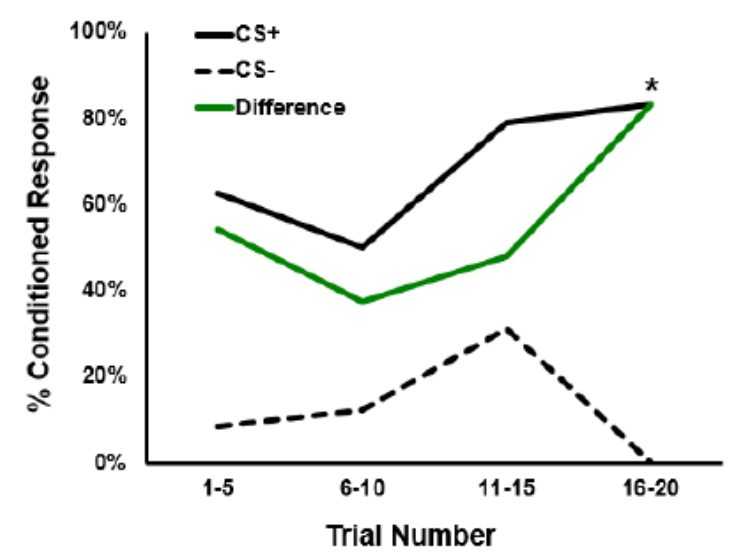

Fig. 7. Mean behavioral performance for the first 20 experiments, the first day of visual signal training for animals in the group. Changes in the response rate to CS + can be seen in the last five recent experiments. * shows significant difference between CS + and CS-. p <.05.

\section{Pure Tones}
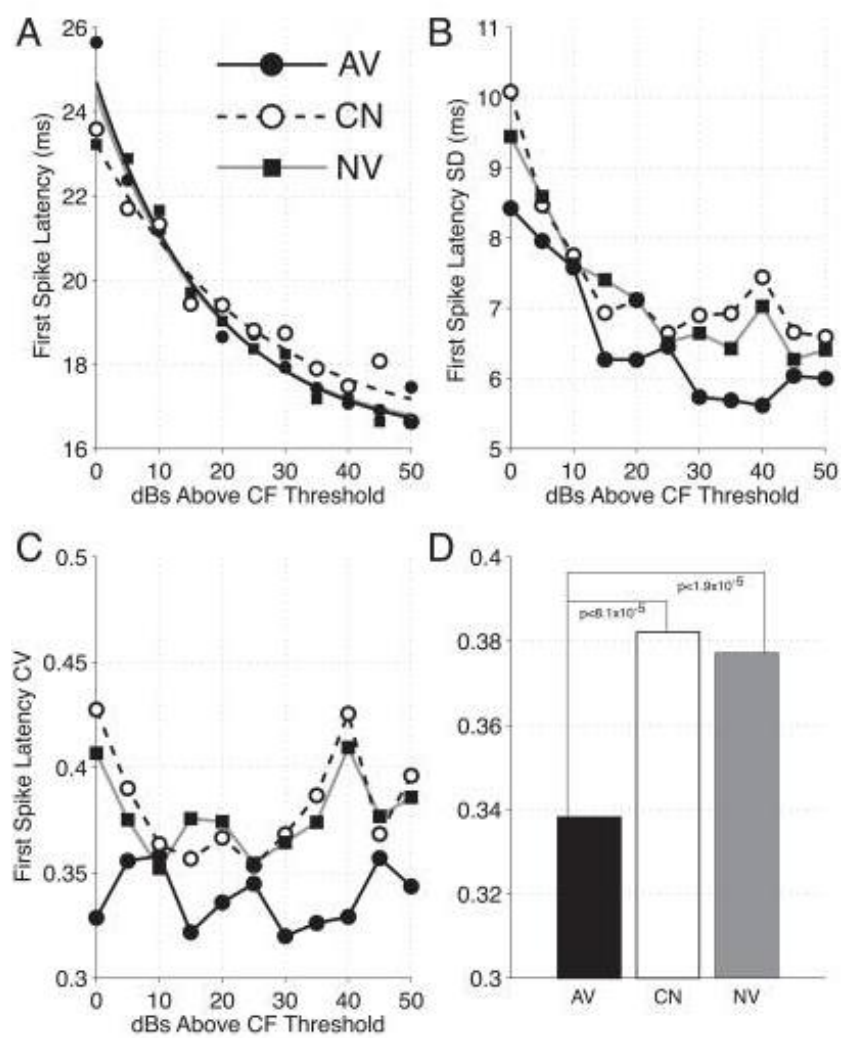

Fig. 8. The influence of behavioral training on the first spikes after purification. (A) Spike time shortage versus stimulation level. Exercise to avoid spike delay 1 did not affect.

A slight and important vary was noted for the 1-spike delay of the $\mathrm{CN}$ group, so that the time delay at the lowest stimulus and slightly higher at the highest stimulus levels were slightly shorter (for both values of $\mathrm{P}<0.05$ ). (B-D) Changes in delay in the first spike against the stimulation level. For all three groups, the standard deviation of spike delays 1 was decreased due to increased stimulus (B). At all levels of mobility, the standard deviation (hence, variable) delay was lower for the AV group. This was clear even when the change was expressed by the coefficient of change (C). When intra-group CVs were collected throughout the stimulant, the AV group was found to be significantly lower than the $\mathrm{CN}$ and $\mathrm{NV}$ groups (for both comparisons, Bonferroni test, $\mathrm{P}$ $<0.05$ ). CV for CN and NV groups was not significantly different (D). AV = solid, $\mathrm{CN}=$ painted, $\mathrm{NV}=$ gray for all graphs. CONCLUSION

This study is in an effort to perform the active avoidance test in rodents, and to extend the work on rabbits by Gabriel et al. In order to create a repeatable paradigm, a visual safety signal was added at different times during the training, in addition to improved animal learning added during training or from the beginning of the training. The results showed that when checking animals that had added visual immunological signal during their training (Switched), a significant increase was found in response to $\mathrm{CS}+$, while only small changes in response to the CS-indicated by the addition of light stimuli Which light stimulus affects CS + response. However, compared with all the groups, the CS-response was higher for people without visual signal (without) more than those trained with switched and with the signal. In addition, data has shown that adding a visual safety signal improves learning and performance in a pro-active discriminatory paradigm. Time-to-measure in animals using the visual immune signal 
significantly decreased, and the percentage of animals that successfully learned this task was added when the visual safety signal was observed. This indicates that the visual safety signal affects the acquisition and maintenance of responses in this work. In addition, this paper shows that the introduced paradigm is used not only in delay mode, but also in tracing air conditioning. The transition to ventilation tracking will allow for further investigation of the memory storage effect in this paradigm.

\section{REFERENCES}

[1] S. M. Metev and V. P. Veiko, Laser Assisted Microtechnology, 2nd ed., R. M. Osgood, Jr., Ed. Berlin, Germany: Springer-Verlag, 1998.

[2] J. Breckling, Ed., The Analysis of Directional Time Series: Applications to Wind Speed and Direction, ser. Lecture Notes in Statistics. Berlin, Germany: Springer, 1989, vol. 61.

[3] S. Zhang, C. Zhu, J. K. O. Sin, and P. K. T. Mok, "A novel ultrathin elevated channel low-temperature poly-Si TFT," IEEE Electron Device Lett., vol. 20, pp. 569-571, Nov. 1999.

[4] M. Wegmuller, J. P. von der Weid, P. Oberson, and N. Gisin, "High resolution fiber distributed measurements with coherent OFDR," in Proc. ECOC'00, 2000, paper 11.3.4, p. 109.

[5] R. E. Sorace, V. S. Reinhardt, and S. A. Vaughn, "High-speed digital-to-RF converter," U.S. Patent 5 668 842, Sep. 16, 1997.

[6] (2007) The IEEE website. [Online]. Available: http://www.ieee.org/

[7] M. Shell. (2007) IEEEtran webpage on CTAN. [Online]. Available: http://www.ctan.org/tex-archive/macros/latex/contrib/IEEEtran/

[8] FLEXChip Signal Processor (MC68175/D), Motorola, 1996.

[9] "PDCA12-70 data sheet," Opto Speed SA, Mezzovico, Switzerland.

A. Karnik, "Performance of TCP congestion control with rate feedback: TCP/ABR and rate adaptive TCP/IP," M. Eng. thesis, Indian Institute of Science, Bangalore, India, Jan. 1999

[10] J. Padhye, V. Firoiu, and D. Towsley, "A stochastic model of TCP Reno congestion avoidance and control," Univ. of Massachusetts, Amherst, MA, CMPSCI Tech. Rep. 99-02, 1999.

[11] Wireless LAN Medium Access Control (MAC) and Physical Layer (PHY) Specification, IEEE Std. 802.11, 1997. 